\title{
A family of models of partially relaxed stellar systems
}

\section{Dynamical properties}

\author{
M. Trenti ${ }^{1}$ and G. Bertin ${ }^{2}$ \\ 1 Scuola Normale Superiore, Piazza dei Cavalieri 7, 56126 Pisa, Italy \\ e-mail: m.trenti@sns.it \\ 2 Dipartimento di Fisica, Università di Milano, via Celoria 16, 20133 Milano, Italy \\ e-mail: Giuseppe.Bertin@unimi.it
}

Received 5 April 2004 / Accepted 27 August 2004

\begin{abstract}
Recently we have found that a family of models of partially relaxed, anisotropic stellar systems, inspired earlier by studies of incomplete violent relaxation, exhibits some interesting thermodynamic properties. Here we present a systematic investigation of its dynamical characteristics, in order to establish the basis for a detailed comparison with simulations of collisionless collapse, planned for a separate paper. For a full comparison with the observations of elliptical galaxies, the models should be extended to allow for the presence a sizable dark halo and of significant rotation. In the spherical limit, the family is characterized by two dimensionless parameters, i.e. $\Psi$, measuring the depth of the galaxy potential, and $v$, defining the form of a third global quantity $Q$, which is argued to be approximately conserved during collisionless collapse (in addition to the total energy and the total number of stars).

The family of models is found to have the following properties. The intrinsic density profile beyond the half-mass radius $r_{M}$ is basically universal and independent of $\Psi$. The projected density profiles are well fitted by the $R^{1 / n}$ law, with $n$ ranging from 2.5 to 8.5 , dependent on $\Psi$, with $n$ close to 4 for concentrated models. All models exhibit radial anisotropy in the pressure tensor, especially in their outer parts, already significant at $r \approx r_{M}$. At fixed values of $v$, models with lower $\Psi$ are more anisotropic; at fixed values of $\Psi$, models with lower $v$ are more concentrated and more anisotropic. When the global amount of anisotropy, measured by $2 K_{\mathrm{r}} / K_{\mathrm{T}}$, is large, the models are unstable with respect to the radial-orbit instability; still, a wide region of parameter space (i.e., sufficiently high values of $\Psi$, for $v>3 / 8$ ) is covered by models that are dynamically stable; for these, the line profiles (line-of-sight velocity distribution) are Gaussian at the 5\% level, with a general trend of positive values of $h_{4}$ at radii larger than the effective radius $R_{\mathrm{e}}$.
\end{abstract}

Key words. stellar dynamics - galaxies: evolution - galaxies: formation - galaxies: kinematics and dynamics galaxies: structure

\section{Introduction}

A simple picture for the incomplete violent relaxation of stellar systems considers the collapse of a dynamically cold cloud of stars or star-clumps initially far from equilibrium. The study of this ideal and relatively simple process, pioneered by important analysis in the 60s (starting with Lynden-Bell 1967) and simulations in the 80s (see van Albada 1982), is still incomplete. A proper understanding of such process is a prerequisite to more ambitious attempts at constructing physically justified models of elliptical galaxies in which the problem of galaxy formation is set in the generally accepted cosmological context of hierarchical clustering.

Before the development of such cosmological scenarios, a first step (in the direction of incorporating in a simple analytical and physically justified framework the clues gathered from simulations of collisionless collapse) had been taken in terms of the so-called $f_{\infty}$ models (Bertin \& Stiavelli 1984). These models are constructed from a distribution function which, in the spherical limit, reduces to $f_{\infty}=A(-E)^{3 / 2} \exp (-a E-$ $c J^{2} / 2$ ), for negative values of $E$, and vanishes for positive values of $E$; here $A, a$, and $c$ are positive constants and $E$ and $J$ denote the specific star energy and angular momentum.

From those earlier investigations, it immediately became clear that the physical clues gathered from the picture of collisionless collapse (i.e., that incomplete violent relaxation leads to systems that are well relaxed in their inner regions, $r \ll r_{M}$, and characterized by radially anisotropic envelopes for $r \gg r_{M}$ ) do not lead uniquely to the $f_{\infty}$ models, but instead identify a wide class of attractive distribution functions of which $f_{\infty}$ represents just one simple and interesting case. At that time, the primary goal of a series of investigations (described, e.g., by Bertin \& Stiavelli 1993) was to test whether the models inspired by studies of collisionless collapse were realistic and thus could serve as a useful tool to interpret the observations. 
Indeed, the $f_{\infty}$ models turned out to "explain" the $R^{1 / 4}$ luminosity law (de Vaucouleurs 1948) and, extended to the twocomponent case, were used successfully to probe the presence and size of dark halos in elliptical galaxies.

Because of the focus on such astronomical applications, the problem of a detailed comparison between the $f_{\infty}$ or other models and the products of collisionless collapse from $N$-body simulations was given lower priority and basically left aside. Yet, it was pointed out that, with respect to the products of numerical simulations of collisionless collapse, the $f_{\infty}$ models had the undesired feature of being too isotropic. Some authors (Merritt et al. 1989) thus suggested that the $f_{\infty}$ models should be extended to and used in the parameter domain where $a<$ 0 ; however, this attempt failed, not only because a proper physical justification was lacking, but especially because such "negative-temperature" models suffer from the opposite difficulty, i.e. they were shown to be so anisotropic that they are violently unstable and bound to evolve on a time scale even smaller than the typical crossing time.

In a recent paper (Bertin \& Trenti 2003) we have revisited the problem of the structure and dynamics of partially relaxed stellar systems starting from a thermodynamic description. In view of the paradigm of the gravothermal catastrophe (see Antonov 1962; Lynden-Bell \& Wood 1968; Katz 1978, 1979, 1980), we found it appropriate to approach the problem in terms of the so-called $f^{(v)}$ models (for a definition, see following Sect. 2); with respect to the $f_{\infty}$ models, the $f^{(v)}$ models follow from a statistical mechanical derivation (Stiavelli \& Bertin 1987) that is formally straightforward, while they are also known to have generally similar and reasonably realistic structural properties. It should be noted that the dynamical properties of the $f^{(v)}$ models had not been studied earlier in detail. Such recent investigation, focusing on the issue of the gravothermal catastrophe, convinced us that the $f^{(v)}$ models do have many attractive features; in particular, these models turn out to have a higher degree of radial pressure anisotropy with respect to the $f_{\infty}$ models, making them more suitable to describe the results of simulations of collisionless collapse. Such encouraging preliminary inspection was the basis for a thorough study that we have thus performed and that we present here.

In this paper we provide a systematic description of the dynamical properties of the $f^{(v)}$ models. In the spherical limit, these models define a two-parameter family, characterized by the dimensionless parameter $\Psi$, measuring the depth of the galaxy potential, and the dimensionless parameter $v$, defining the form of a third global quantity $Q$ (which is argued to be approximately conserved during collisionless collapse, in addition to the total energy and the total number of stars; see Sect. 2 for the relevant definitions). In Sect. 3 we present intrinsic and projected density profiles and fit the latter structural characteristics in terms of the $R^{1 / n}$ law (Sersic 1968). We then proceed to illustrate in detail their phase space properties, in particular by calculating the relevant pressure tensor profiles, the pressure anisotropy content, the projected velocity dispersion profiles, the line profiles (line-of-sight velocity distribution), and the relevant phase space densities $N\left(E, J^{2}\right)$ and $N(E)$ (Sect. 4). In Sect. 5 we examine the stability of the models with respect to the radial orbit instability, not only by inspection of well-known global anisotropy indicators, but also with the aid of a number of $N$-body simulations. In Sect. 6 we perform a first test of the viability of these models by applying them to the observed properties of NGC 3379, a galaxy that is characterized by lack of significant rotation and is likely to possess only small amounts of dark matter. The conclusions are drawn in Sect. 7.

This paper also sets the basis for a detailed comparison between the $f^{(v)}$ family of models and the products of collisionless collapse resulting from $N$-body simulations, to be presented in a follow-up paper. For a full comparison with the observations of elliptical galaxies, the models should be extended to allow for the presence of a sizable dark halo and of significant rotation.

\subsection{Models of galactic structure: Physical outlook}

The need for anisotropic models to describe elliptical galaxies can be traced back to the strong empirical arguments that come from the observations of non-spherical geometry in the absence of significant rotation (e.g., see the discussion provided by Bertin \& Stiavelli 1993, and references therein); to be sure, direct evidence for pressure anisotropy in round systems is not easy to obtain, because so far the observed line profiles show only modest deviations from a Gaussian (e.g., see Gerhard et al. 2001).

The main goal of this paper is to study the properties of a given specific dynamical context able to provide physical justification for the existence of anisotropic equilibria, that is the dynamical framework of collisionless collapse. The process of collisionless collapse, together with its accompanying mechanism of incomplete violent relaxation, is one (but not the only) element that is expected to play an important role in the formation of stellar systems. In spite of the many papers that have addressed issues related to such dynamical context (in addition to the papers cited earlier in the Introduction, e.g. see Shu 1978, 1987; Voglis 1994; Hjorth \& Madsen 1995), it is not yet clear whether an analytically tractable distribution function, or family of distribution functions, can be assigned to the products of such collisionless collapse. This paper tries to provide a contribution to this problem. At the same time, we still need to establish how far the properties of such products, often studied in a simplified one-component picture, would be from those of observed stellar systems, and in which way.

An enormous amount of work has focused and is currently focusing on the demands (on galactic structure) from the cosmological context. In particular, this has led astronomers to look for the presence, in observed objects, of cuspy density distributions of dark matter, following a universal profile suggested by cosmological simulations (see Navarro et al. 1997; Moore et al. 1998; Ghigna et al. 2000). Other studies have addressed the issue of the establishment of the galaxy scaling laws (such as the Fundamental Plane for elliptical galaxies; e.g., see Ghigna et al. 2000; Lanzoni et al. 2004) in the accepted cosmological scenario. These demands are beyond the scope of the present paper, but should eventually be faced, as 
a point of contact between dynamical investigations of individual galaxies and studies of the evolving universe from which they were formed.

In this respect, a point to be noted, to avoid unnecessary confusion about the aims of purely dynamical studies, is the following. A priori, studies of collisionless collapse within the line of research adopted in this paper have nothing to say about some important issues such as the establishment of the Fundamental Plane (but see Gonzalez-Garcia \& van Albada 2003; Nipoti et al. 2003), because the relevant scaling laws depend on physics that goes beyond pure dynamics, which is inherently scale-free. In turn, pure dynamical studies can try to explain why galaxies prefer the $R^{1 / 4}$ law, which is a structural property, instead of just accepting it as an empirical fact (as often done in a number of otherwise important astrophysical studies).

Thus we would like to emphasize that this paper represents only one step in the direction of a comparison with the observations. To deal fruitfully with the presence of dark matter and other important ingredients (such as significant rotation and the possible presence of an additional disk component), one first has to master the properties of one-component models, which, as shown in this paper, turn out to exhibit a variety of interesting dynamical properties. In fact, it is rewarding and a priori unexpected to find that, as a result of a simple conjecture about the way to characterize incomplete violent relaxation (the addition of $Q$ to the natural constraints under which Boltzmann entropy is extremized; see Sect. 2), one-component spherical models are identified able to fit products of $N$-body simulations over nine orders of magnitude in density (see Bertin \& Trenti 2004) and, at the same time, the observed photometric profile (over about ten magnitudes) and the inner kinematic profile (inside $R_{\mathrm{e}}$ ) of the best studied elliptical galaxy (see Sect. 6). Therefore, in spite of its incompleteness, the stage reached so far is definitely interesting from the physical point of view. Discrepancies with respect to the observations play the welcome role of providing concrete indications about the role of the ingredients that are a priori ignored by the purely dynamical and highly simplified picture considered in this paper.

\section{Model construction and the relevant parameter space}

In the spherically symmetric limit, in order to allow for the possibility that a stellar system is only partially relaxed, one may extremize the Boltzmann entropy $S=-\int f \ln f \mathrm{~d}^{3} x \mathrm{~d}^{3} w$ under the constraint that the total energy $E_{\text {tot }}=(1 / 3) \int E f \mathrm{~d}^{3} x \mathrm{~d}^{3} w$, the total mass $M=\int f \mathrm{~d}^{3} x \mathrm{~d}^{3} w$, and the additional quantity

$Q=\int J^{v}|E|^{-3 v / 4} f \mathrm{~d}^{3} x \mathrm{~d}^{3} w$

are constant (Stiavelli \& Bertin 1987). Here $E=w^{2} / 2+\Phi$ and $J^{2}=|\boldsymbol{r} \times \boldsymbol{w}|^{2}$ represent the specific energy and the specific angular momentum square of a single star subject to a spherically symmetric mean potential $\Phi(r)$. (We have decided to use the symbol $w$, instead of $v$, for the velocity variable so as to avoid confusion with the symbol $v$.) If only $E_{\text {tot }}$ and $M$ were kept fixed, the extremization process would lead to a Maxwellian, that is to an isothermal and isotropic distribution function, appropriate for a fully relaxed system. Some arguments have been provided as to why a quantity such as $Q$ should be, at least approximately, conserved (Stiavelli \& Bertin 1987) and those will not be repeated here. In any case, the conservation of $Q$ should be taken as a conjecture. In a follow-up paper (Trenti et al. in preparation) we will discuss this problem further by examining a set of collapse simulations, for which the conservation of $Q$ will be tested.

Such extremization leads to the following expression for the $f^{(v)}$ distribution function:

$f^{(v)}=A \exp \left[-a E-d\left(\frac{J^{2}}{|E|^{3 / 2}}\right)^{v / 2}\right]$,

where $v, a, A$, and $d$ are positive real constants. This set of constants provides two dimensional scales (for example a mass scale $M_{\text {scale }}=A a^{-9 / 4} d^{-3 / v}$ and a reference radius $R_{\text {scale }}=$ $\left.a^{-1 / 4} d^{-1 / v}\right)$ and two dimensionless parameters. For the latter two quantities, we may refer to $v$ and $\gamma=a d^{2 / v} /(4 \pi G A)$. The distribution function is taken to vanish for unbound orbits, that is for $E>0$.

The two-parameter family of models is then constructed by solving the Poisson equation:

$\nabla^{2} \Phi(\boldsymbol{r})=4 \pi G \int f^{(v)}(\boldsymbol{r}, \boldsymbol{w}) \mathrm{d}^{3} \boldsymbol{w}$,

for the potential $\Phi(r)$. As briefly described by Bertin \& Trenti (2003), the solution is obtained numerically after the equation has been cast in dimensionless form:

$\frac{1}{\hat{r}^{2}} \frac{\mathrm{d}}{\mathrm{d} \hat{r}} \hat{r}^{2} \frac{\mathrm{d}}{\mathrm{d} \hat{r}} \hat{\Phi}=\frac{1}{\gamma} \hat{\rho}(\hat{r}, \hat{\Phi})$,

with $\hat{r}=r / R_{\text {scale }}$ and $\hat{\Phi}=a \Phi$ (for the adopted scaling, see the Appendix). The Poisson equation is integrated with "initial" conditions $\mathrm{d} \hat{\Phi} / \mathrm{d} r=0$ and $\hat{\Phi}=-\Psi$ at $r=0$ and the parameter $\gamma$ is determined as an eigenvalue, $\gamma=\gamma(\Psi)$, in order to satisfy the condition of Keplerian decay $(\hat{\Phi} \sim-1 / \hat{r})$ at large radii. The general behavior of the function $\gamma(\Psi)$ is similar to that of the corresponding function for the $f_{\infty}$ models; after some oscillations, $\gamma$ tends to a "plateau" at large values of $\Psi$ (as indicated by Fig. 1).

We have explored the parameter space $(v, \Psi)$ by means of an equally spaced grid from $v=3 / 8$ to $v=1$ at steps of $1 / 8$, and from $\Psi=2$ to $\Psi=13$ at steps of 0.2 . A given model will be denoted by the values of the two parameters $(v ; \Psi)$ in parentheses.

For given $(v, \Psi)$, once the solution for the potential $\Phi(r)$ is obtained, it can be inserted in the expression of $f^{(v)}$, from which all the intrinsic and observable profiles and properties of the model can be calculated. In particular the density $\rho(r)$ and the anisotropy profile $\alpha(r)$, defined as $\alpha(r)=2-\left(\left\langle w_{\theta}^{2}\right\rangle+\right.$ $\left.\left\langle w_{\phi}^{2}\right\rangle\right) /\left\langle w_{\mathrm{r}}^{2}\right\rangle$, require the evaluation of a double integral over the velocity space (see also Bertin \& Trenti 2003).

The problem of constructing the line profiles $F(w, R)$ for a given distribution function of the form $f\left(E, J^{2}\right)$ is often discussed in the literature (see Gerhard 1991, 1993; 


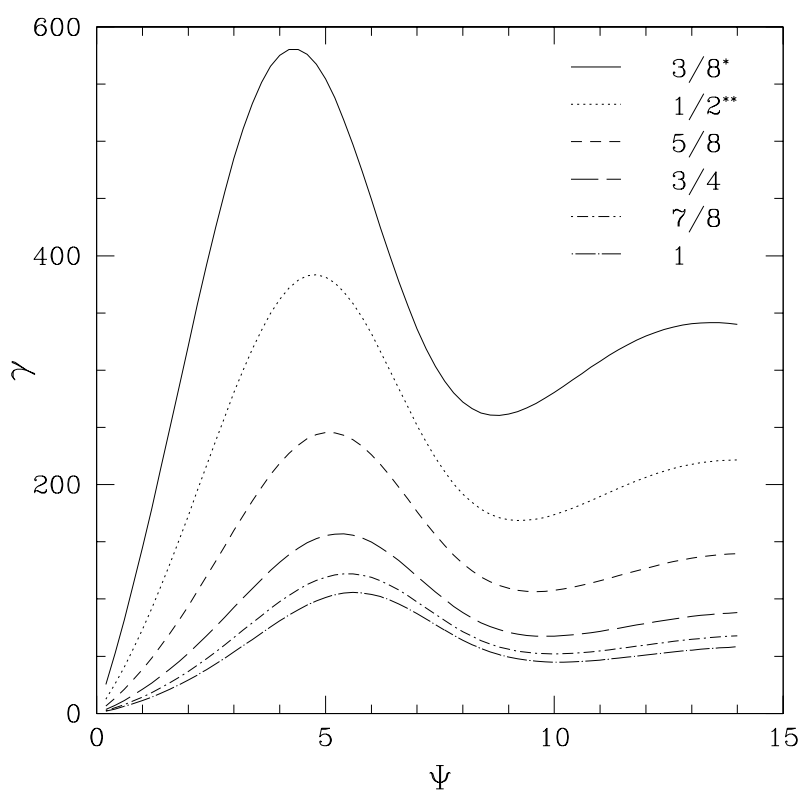

Fig. 1. Relation $\gamma(\Psi)$ for the $f^{(v)}$ family of models, for selected values of $v$. To fit the adopted frame, the $\gamma$ values corresponding to $v=3 / 8$ have been multiplied by a factor $1 / 6$ and the ones corresponding to $v=1 / 2$ by a factor $2 / 3$.

Carollo et al. 1995). The calculation requires the evaluation of a triple integral (two integrations over the velocity space orthogonal to the line of sight and one over the radial coordinate along the line of sight), which we have performed numerically with the same adaptive algorithm used to compute the density (Berntsen et al. 1991). In practice, we have followed the procedure described by Gerhard (1991). The calculation of the quantities $\sigma_{\text {proj }}$ and $h_{4}$ is then performed as described at the end of Sect. 4.3, where they are introduced and defined.

\subsection{The parameter $\Psi$ and the density concentration}

In the following we will often refer to the parameter $\Psi$ as the concentration parameter. Strictly speaking, such term is justified only at relatively large values of $\Psi(\Psi \gtrsim 5)$.

A more intuitive measure of the central concentration of a model is given by the ratio $\rho(0) / \rho\left(r_{M}\right)$ of the central density to the value of the density attained at the half-mass radius $r_{M}$. As illustrated in Fig. 2, this ratio is a monotonic increasing function of $\Psi$ only beyond a minimum at $\Psi \approx 4.5$.

\section{Density profiles}

In this section we start with the discussion of the properties of the density distribution.

\subsection{The intrinsic density profile}

In the outer parts, i.e. at radii such that $r \gtrsim r_{M}$, the density profile (Fig. 3) is basically the same for all models of the $f^{(v)}$ family. In fact, the dimensionless density associated with

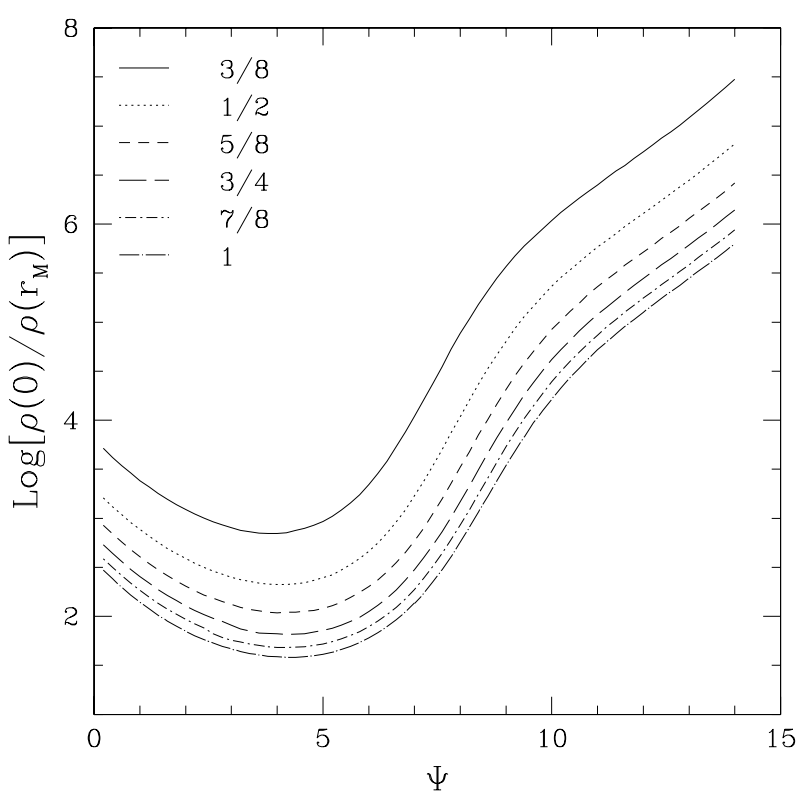

Fig. 2. Density contrast between the center and the half-mass radius along the equilibrium sequence for different values of $v$. The solid line refers to $v=3 / 8$; at fixed $\Psi$, models with higher values of $v$ are less concentrated.

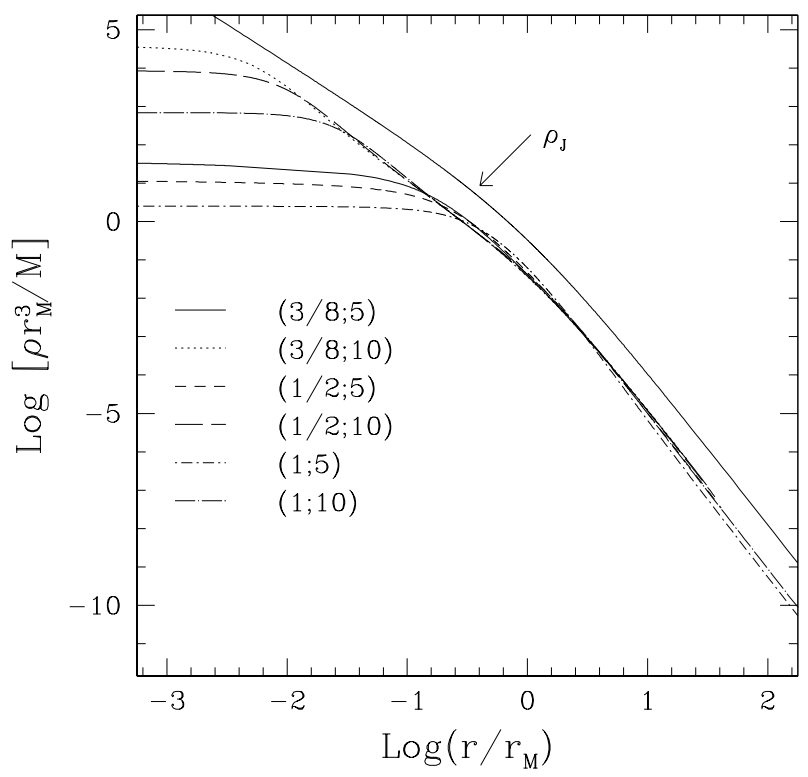

Fig. 3. Density profile of some representative $f^{(v)}$ models, with $v=$ $3 / 8,1 / 2$, and 1 , and $\Psi=5$ and 10 . The most concentrated model corresponds to $(3 / 8 ; 10)$ and the least concentrated to $(1 ; 5)$. If the scales are fixed so that $r_{M}=10 \mathrm{kpc}$ and $M=10^{11} M_{\odot}$, the units for the density are $10^{-1} M_{\odot} / \mathrm{pc}^{3}$. The density profiles overlap in the outer parts, beyond a radius that becomes smaller and smaller as $\Psi$ increases. In the plot we also record the $\rho_{J}$ profile, shown here on an arbitrary scale for a convenient comparison.

the $f^{(v)}$ distribution function can be expressed, at large values of $r$, as:

$\hat{\rho}(\hat{r})=\frac{3 \pi^{2}}{2 \sqrt{2}} \frac{\Gamma(2 / v)}{v} \frac{1}{\hat{r}^{2}}\left(\hat{\Phi}^{2}(\hat{r})+O\left(\hat{\Phi}^{3}\right)\right)$,

where $\Gamma(x)$ is the standard Gamma function Abramowitz \& Stegun (1965). Curiously, the $1 / r^{4}$ behavior is found to start 
from well inside the main body of the system, not too far beyond $r_{M}$, while the density distribution of the inner half of the system is similar to that of an isothermal sphere. A power-law of the form $r^{-3.2}$ fits reasonably well the profiles in the transition region from $r_{M}$ to $3 r_{M}$.

As the concentration parameter $\Psi$ increases, the point where the density profiles merge into a profile common to all the models sets in at smaller and smaller radii, so that for increasing values of $\Psi$ the models appear to converge toward a common (singular) model with a central cusp. In these respects, the general behavior of the intrinsic density profiles is similar to that of the $f_{\infty}$ models. Therefore, the behavior of concentrated models is well captured by the following simple formula (Jaffe 1983):

$\hat{\rho}_{J}(\hat{r})=\frac{1}{\hat{r}^{2}} \frac{1}{(1+\hat{r})^{2}}$,

as shown in Fig. 3. Here, and in the remaining part of this subsection, a hat symbol denotes that a quantity is expressed in a suitable dimensionless form, obviously with no reference to the scaling procedure described after Eq. (2).

In turn, low $-\Psi$ models are characterized by a prominent core. In fact, some models have density profiles close to that of the isochrone model (Hénon 1959) or of the so-called perfect sphere $\left(\hat{\rho}_{\mathrm{p}}=\left(1+\hat{r}^{2}\right)^{-2}\right)$. The core may be even represented by the density profile of a Plummer sphere (Plummer 1915),

$\hat{\rho}_{\mathrm{Pl}}(r)=\frac{1}{\left(1+\hat{r}^{2}\right)^{5 / 2}}$.

These density profiles are mentioned to better illustrate the properties of the mass distribution of the $f^{(v)}$ models in terms of well-known profiles. For a discussion of the merits and limitations of physically based models (such as the $f^{(v)}$ models) with respect to other models constructed on the basis of analytical convenience (e.g., see Merritt 1985; Hernquist 1990), the reader is referred to the review article by Bertin \& Stiavelli (1993). On the other hand, in relation to phase-space properties, we should emphasize the following point. As will be clear from Sect. 4, in the approach adopted in this paper (in which the distribution function is constructed from physical arguments) the final velocity dispersion and pressure anisotropy profiles cannot be set independently, but follow from the self-consistent solution. In other words, all the "observable" profiles can be seen as consequences of the physical framework considered. This is in sharp contrast with other modeling procedures that are descriptive, rather than predictive (e.g., see Merritt 1985).

\subsection{The projected density profile}

We have then proceeded to compute a library of projected density profiles (which may be compared to observed luminosity profiles, under the assumption of a constant $M / L$ ratio). A first way to characterize these profiles is to fit them with the $R^{1 / n}$ law (Sersic 1968). Such fit has been performed over a very wide radial range, from $0.1 R_{\mathrm{e}}$ to $10 R_{\mathrm{e}}$. It shows that the $f^{(v)}$ family is well represented by the $R^{1 / n}$ law, with the index $n$ ranging from 2.5 to 8.5 (see Fig. 4 ; the slightly bumpy behavior

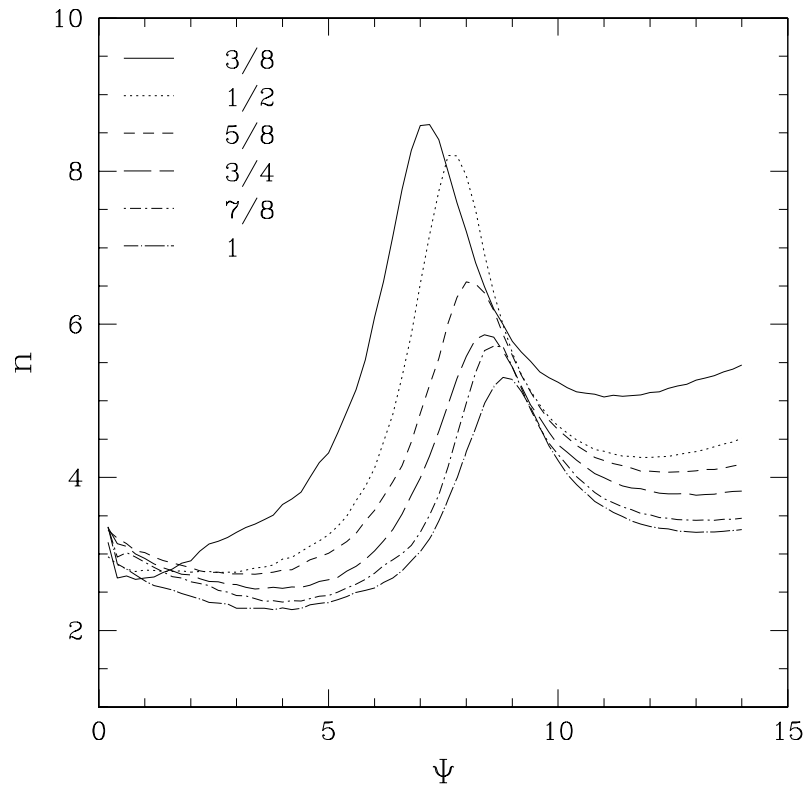

Fig. 4. Best fit value of the index $n$ (of the $R^{1 / n}$ law) associated with the projected density profile of the $f^{(v)}$ models, for selected values of $v$.

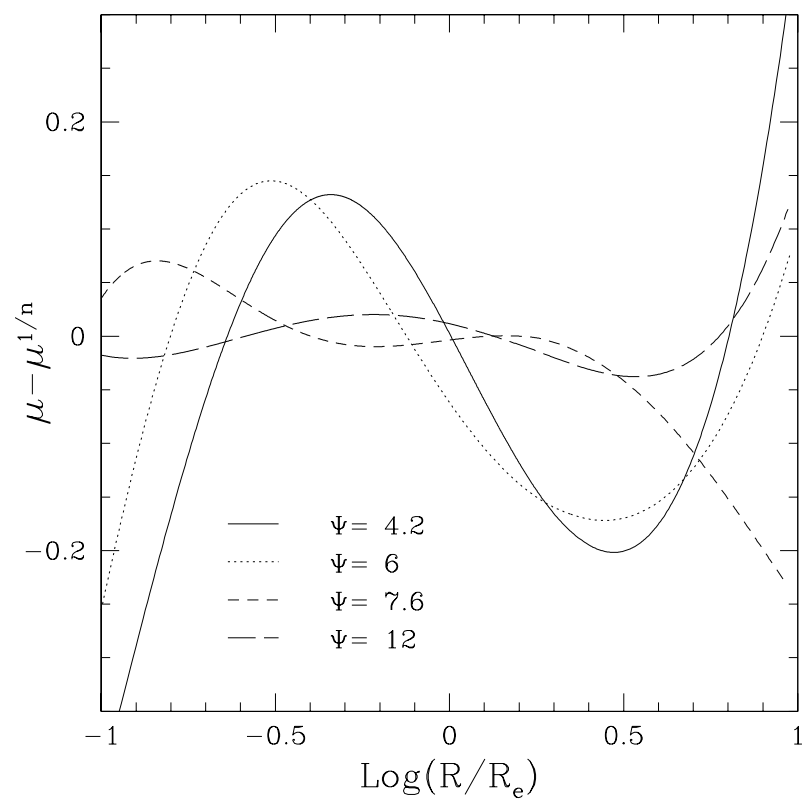

Fig. 5. Residuals $\mu-\mu^{1 / n}$, in magnitudes, obtained by fitting the $R^{1 / n}$ law to the projected density profiles of the $f^{(v)}$ models for $v=1 / 2$ and selected values of $\Psi$. The quality of the fit is excellent for concentrated models $(\Psi>7)$.

of the $v=3 / 8$ curve just reminds us of the uncertainties associated with the best-fit determination of $n$ ). The $n=4$ behavior, characteristic of the de Vaucouleurs law (de Vaucouleurs 1948 ), is mostly associated with concentrated high- $\Psi$ models, but we note that many intermediate- $\Psi$ models also have the same structural property. The residuals from the $R^{1 / n}$ best fit (see Fig. 5) are typically within 0.05 mag for concentrated models, while at low values of $\Psi$ they are within 0.2 mag; the general behavior can be compared with that of the $f_{\infty}$ models (see Fig. A.1 in Bertin et al. 2002). 


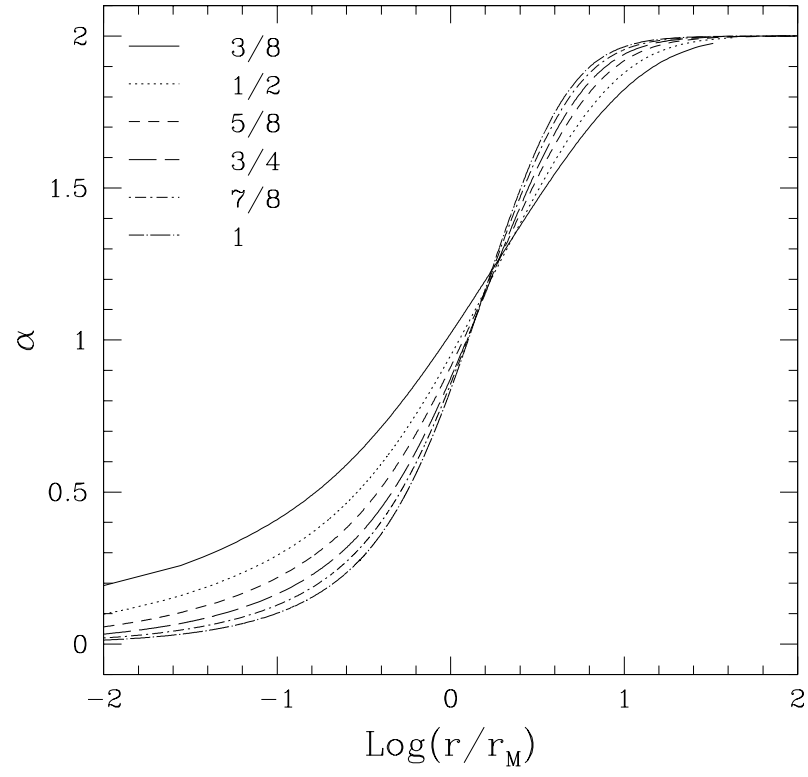

Fig. 6. Anisotropy profiles $\alpha(r)$ of $\Psi=5$ models for different values of $v$.

\section{Phase space properties}

Here we describe the properties of the $f^{(v)}$ models related to the velocity space.

\subsection{Pressure anisotropy profiles and global anisotropy indicators}

The pressure anisotropy of the models can be described by means of the anisotropy profile $\alpha(r)$, defined as $\alpha(r)=2-$ $\left(\left\langle w_{\theta}^{2}\right\rangle+\left\langle w_{\phi}^{2}\right\rangle\right) /\left\langle w_{\mathrm{r}}^{2}\right\rangle$. This function, illustrated in Fig. 6, shows that the cores are approximately isotropic and that in the outer parts the pressure is mostly in the radial direction, in line with the qualitative expectations of the violent relaxation scenario (Lynden-Bell 1967). Higher values of $v$ are associated with a sharper transition from central isotropy to radial anisotropy.

The degree of anisotropy globally present in our models can be characterized in terms of the ratio $2 K_{\mathrm{r}} / K_{\mathrm{T}}$, where $K_{\mathrm{r}}$ is the total kinetic energy associated with the radial degree of freedom, and $K_{\mathrm{T}}$ the corresponding quantity related to the two tangential directions. All models present an excess of kinetic energy in the radial direction, as illustrated in Fig. 7. The ratio $2 K_{\mathrm{r}} / K_{\mathrm{T}}$ is greater than $\approx 1.3$ over the whole sequence and becomes larger than 2 in the low- $\Psi$ region. As a general trend, at fixed $\Psi, f^{(v)}$ models with higher values of $v$ are more isotropic.

In addition to $2 K_{\mathrm{r}} / K_{\mathrm{T}}$, as a global anisotropy indicator we can also refer to the parameter $r_{\alpha} / r_{M}$ (see Fig. 7); here the radius $r_{\alpha}$ denotes the anisotropy radius, defined from the relation $\alpha\left(r_{\alpha}\right)=1$. In contrast with the $f_{\infty}$ models, for which $r_{\alpha} \approx 3 r_{M}$ for relatively large values of $\Psi$, here we find that concentrated models are characterized by $r_{\alpha} \approx r_{M}$.

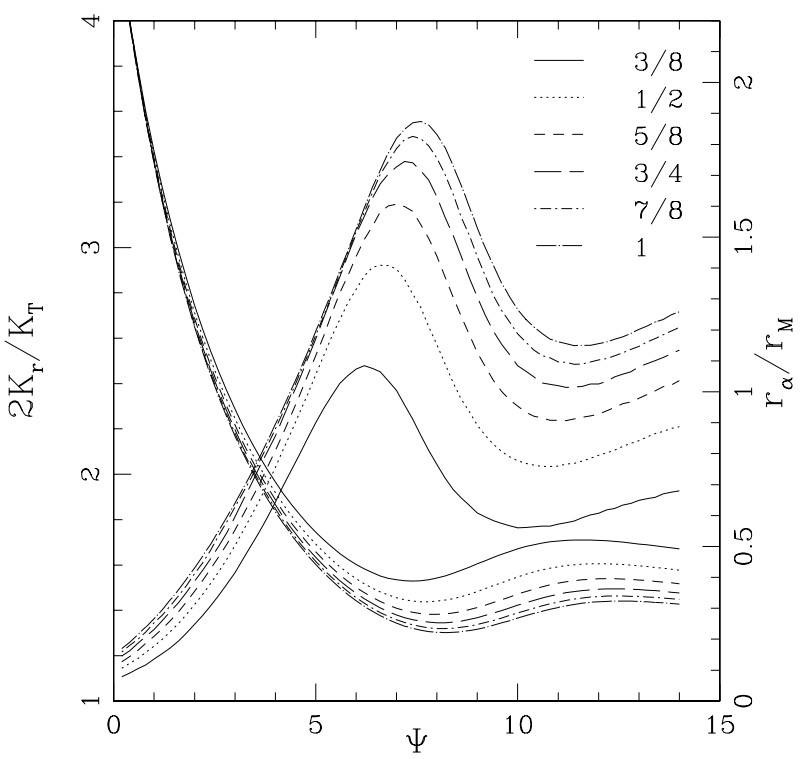

Fig. 7. Global anisotropy of the $f^{(v)}$ models shown in terms of the parameter $2 K_{\mathrm{r}} / K_{\mathrm{T}}$ (group of curves starting from the top left) and of the ratio $r_{\alpha} / r_{M}$ (anisotropy radius to half-mass radius; group of curves starting from the bottom left) as a function of $\Psi$. For low values of $\Psi$ the global anisotropy $2 K_{\mathrm{r}} / K_{\mathrm{T}}$ is basically independent of $v$. At fixed value of $\Psi$ the global anisotropy decreases with $v$.

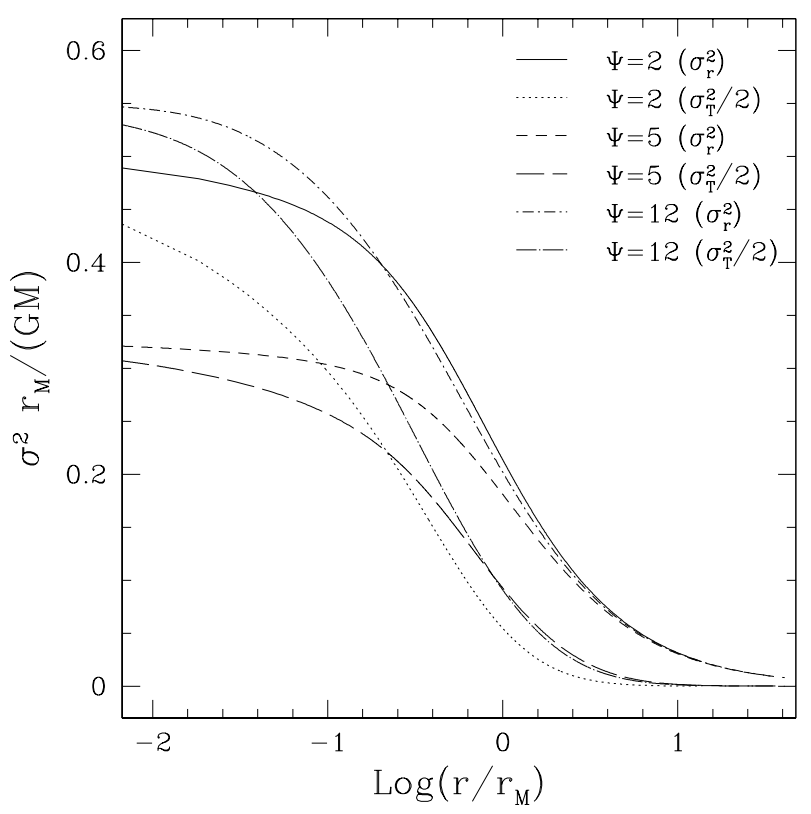

Fig. 8. Intrinsic "pressure" profiles $\left(\sigma_{\mathrm{T}}^{2} / 2=\left(\left\langle w_{\theta}^{2}\right\rangle+\left\langle w_{\phi}^{2}\right\rangle\right) / 2\right.$ and $\sigma_{\mathrm{r}}^{2}=$ $\left.\left\langle w_{\mathrm{r}}^{2}\right\rangle\right)$ for selected $f^{(v)}$ models with $v=1 / 2$.

\subsection{Velocity dispersion and projected velocity dispersion profiles}

As we have seen, the models can be characterized by significant "pressure" anisotropy. This can be illustrated directly by the intrinsic velocity dispersion profiles, for which one may find significant differences between the tangential $\sigma_{\mathrm{T}}^{2} / 2$ and the radial dispersion $\sigma_{\mathrm{r}}^{2}$ as far in as $r \approx 0.1 r_{M}$ (see Fig. 8). These velocity space properties, in combination with the density distribution, give rise to the projected velocity dispersion profiles 


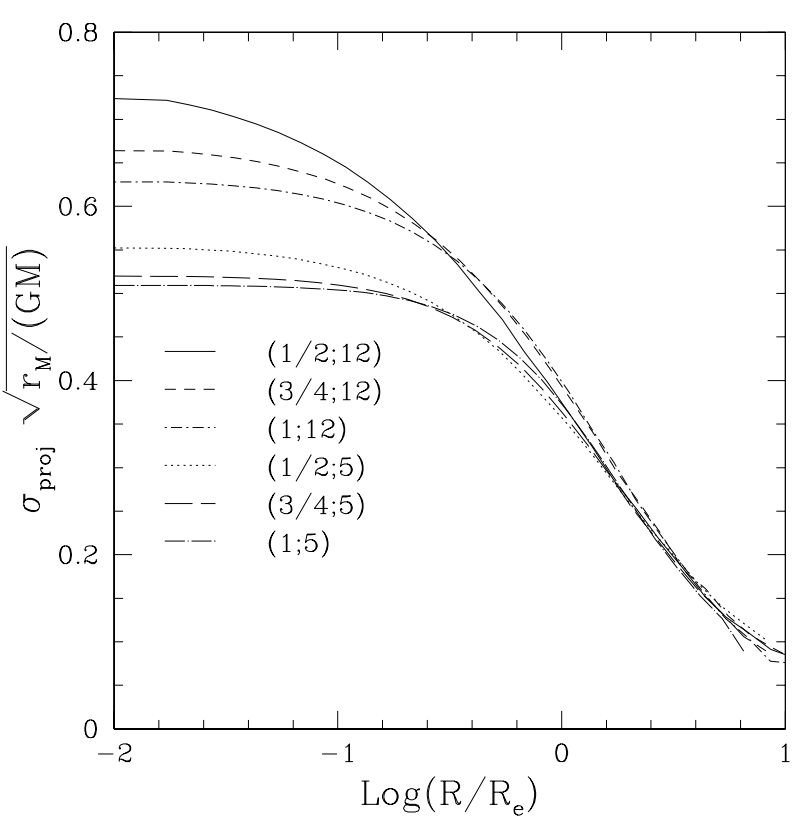

Fig. 9. Projected velocity dispersion profile for selected $f^{(v)}$ models. If the scales are fixed so that $r_{M}=10 \mathrm{kpc}$ and $M=10^{11} M_{\odot}$, the velocity dispersion $\sigma_{\text {proj }}$ is given in units of $207.4 \mathrm{~km} \mathrm{~s}^{-1}$.

(calculated from the line profiles, as described in the next subsection), which may eventually be compared with the observed kinematical profiles; some projected velocity dispersion profiles are shown in Fig. 9.

\subsection{Line profiles}

Pressure anisotropy can affect the shape of the velocity distribution integrated along a given direction, which can be tested observationally by studying the profiles of the lines used to determine the observed velocity dispersion. In this context, since observational limitations prevent us from obtaining accurate measurements of line profiles, comparisons between data and models are often carried out in terms of certain shape parameters, which measure the deviations from a Gaussian profile (e.g., see Gerhard 1991; de Zeeuw et al. 2002). In view of this approach, we have first computed the line profiles for a number of models, following the procedure described in Sect. 2 and then we have extracted from them the related value of the $h_{4}$ parameter (being non rotating and spherically symmetric, the $f^{(v)}$ models are associated with line profiles characterized by vanishing $h_{3}$ ).

To extract the velocity dispersion $\sigma_{\text {proj }}$ and the value $h_{4}$ we fit the line profile $F(w, R)$, at fixed $R$, with a Gaussian corrected by a fourth order Gauss-Hermite polynomial Abramowitz \& Stegun (1965):

$$
\begin{aligned}
F(w, R)= & F_{0} \exp \left[-\left(w / \sigma_{\text {proj }}\right)^{2} / 2\right] \\
& \times\left\{1+h_{4}\left[12-48\left(\frac{w}{\sigma_{\text {proj }}}\right)^{2}+16\left(\frac{w}{\sigma_{\text {proj }}}\right)^{4}\right]\right\},
\end{aligned}
$$

where $F_{0}, \sigma_{\text {proj }}$, and $h_{4}$ are free parameters. The fit has been performed with a Simulated Annealing method (Press et al. 1992).

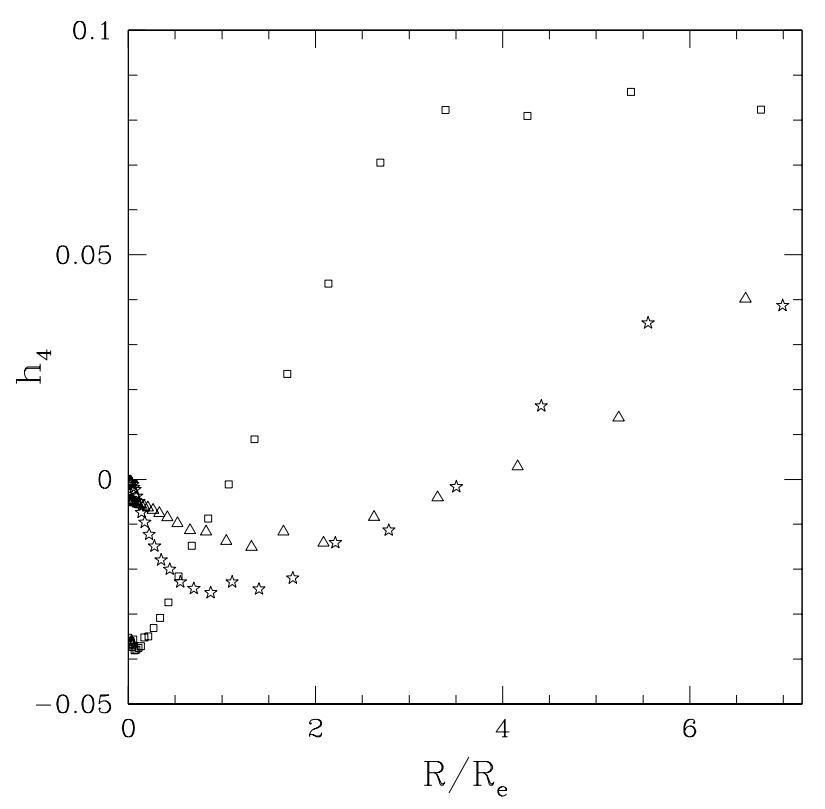

Fig. 10. Profiles of the $h_{4}$ parameter for selected $f^{(v)}$ models with $v=$ $3 / 4$ and different values of $\Psi$ (the $\Psi=2$ model is denoted by squares, $\Psi=6$ by triangles, and $\Psi=10$ by stars).

In general, the deviations from a Gaussian are modest, $\approx 5 \%$, and reduce to within $1 \%$ for $R \lessgtr R_{\mathrm{e}}$. The $h_{4}$ parameter takes on slightly negative values at the center of the system and then becomes positive in the outer parts (see Fig. 10), in line with the results found by Gerhard (1991).

\subsection{The phase space densities $N\left(E, J^{2}\right)$ and $N(E)$}

In view of a comparison with $N$-body simulations, a physically interesting way to characterize the phase space properties of the $f^{(v)}$ models is in terms of the $N\left(E, J^{2}\right)$ and $N(E)$ densities, defined in such a way that $M=\int N(E) \mathrm{d} E=\int N\left(E, J^{2}\right) \mathrm{d} E \mathrm{~d} J^{2}$. Therefore, the relation between $f^{(v)}\left(E, J^{2}\right)$ and the phase space density $N\left(E, J^{2}\right)$ is given by the Jacobian of the transformation from $\mathrm{d}^{3} x \mathrm{~d}^{3} w$ to $\mathrm{d} E \mathrm{~d} J^{2}$ :

$N\left(E, J^{2}\right)=\frac{2 \pi f^{(v)}\left(E, J^{2}\right)}{\Omega_{\mathrm{r}}\left(E, J^{2}\right)}$,

where $\Omega_{\mathrm{r}}\left(E, J^{2}\right)$ is the radial frequency of stellar orbits in the given potential $\Phi(r)$. At variance with the $f_{\infty}$ models, the $f^{(v)}$ models exhibit a singular behavior of $N\left(E, J^{2}\right)$ near the origin in the $\mathrm{d} E \mathrm{~d} J^{2}$ phase space (see Fig. 11).

\section{Stability}

The study of the stability of the $f^{(v)}$ models could be approached either through a linear modal analysis (see Bertin et al. 1994) or by means of $N$-body simulations. Here we prefer the latter approach, starting from initial conditions obtained by sampling the relevant distribution functions with Monte Carlo techniques. 


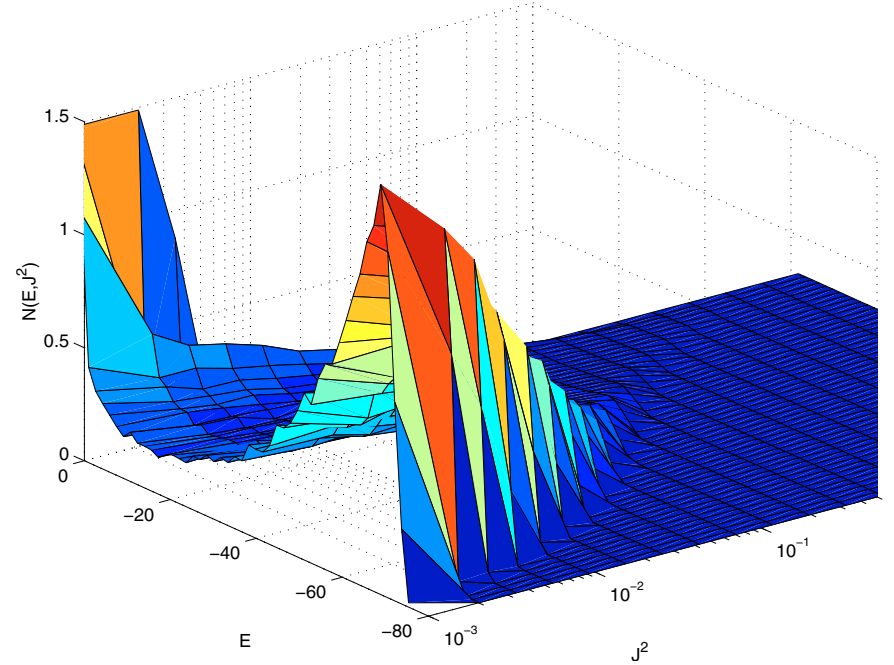

Fig. 11. The phase space density $N\left(E, J^{2}\right)$ for the $(1 / 2 ; 6.2) f^{(v)}$ model. The graph has been obtained by a Monte Carlo sampling of the distribution function with $2 \times 10^{6}$ points.

\subsection{General conditions for the onset of the radial orbit instability}

A simple criterion for the onset of the radial orbit instability states that instability occurs for systems with $2 K_{\mathrm{r}} / K_{\mathrm{T}} \gtrsim$ 1.75 (Polyachenko \& Shukhman 1981; see also Fridman \& Polyachenko 1984). We thus note that models with $\Psi \lesssim 4$ should be unstable, in a way that is basically independent of $v$ (cf. Fig. 7).

\subsection{Simulations of stable and unstable configurations}

We have simulated the evolution of different $f^{(v)}$ models by means of an improved version (see Trenti 2004) of a code written originally by van Albada \& van Gorkom (1977; see also van Albada 1982). The evolution of the system is collisionless because the simulation particles interact with one another via a mean field computed with Fourier techniques; the mean density associated with the particle distribution is expanded in spherical harmonics with contributions up to $l=4$ (the code can handle contributions up to $l=6$ ). This choice of code is well suited for our problem, since its performance has been tested to be high for quasi-equilibrium conditions and for smooth spherically symmetric spatial distributions. To further check our results, we have run a comparison simulation with the tree code of Londrillo (see Londrillo et al. 2003) for the $(1 ; 3.2)$ model and found no significant differences.

In order to sample points from the $f^{(v)}$ distribution function and thus to create the initial conditions needed to study the stability of the $f^{(v)}$ models, we have used an exact inversion of the cumulative mass profile and a two-dimensional hit/miss method in the $\left(w_{\mathrm{r}}, w_{\perp}\right)$ velocity space. The angles needed to complete the assignment of the position and velocity vectors of the simulation particles are generated randomly. In principle, the models extend out to infinity. In practice, we set a cut-off radius $r_{\text {cut }} \approx 50 r_{M}$, and we rescale the mass profile in such a way that $M\left(r_{\text {cut }}\right)=M$. The introduced mass "loss" is generally less than $1 \%$.

Most simulations have been run with $2 \times 10^{5}$ particles. If we refer to a system with total mass $M=10^{11} M_{\odot}$ and half-mass radius $r_{M}=5 \mathrm{kpc}$, the evolution is followed from $t=0$ up to $t=t_{\text {end }}=2$ Gyr. This corresponds to more than $20 t_{\mathrm{d}}$, with the natural dynamical time defined as $t_{\mathrm{d}}=G M^{5 / 2} /(2 K)^{3 / 2}$ (here $K=K_{\mathrm{r}}+K_{\mathrm{T}}$ is the total kinetic energy); in fact, for the range of $(v ; \Psi)$ considered and the above scales for $M$ and $r_{M}$, we have $t_{\mathrm{d}} \approx(0.65-0.80) \times 10^{8}$ yr. At $t \gtrsim 10 t_{\mathrm{d}}$ the system, even when it has initially evolved because of initial unstable conditions, settles down into an approximate equilibrium state.

The total energy is typically conserved within $10^{-5}$ over one dynamical time. The models are non-rotating; the total angular momentum remains within $10^{-4}$ around the small value generated in the initialization process.

To quantify the effects of the radial orbit instability, we have especially focused on the evolution of the central density concentration, of the shape of the inertia ellipsoid, computed on the basis of the distribution of the simulation particles within a sphere of radius $3 r_{M}$, and of the global anisotropy $2 K_{\mathrm{r}} / K_{\mathrm{T}}$ ratio. The end-products of unstable initial conditions are characterized by a final prolate state (with axial ratios $a_{2} / a_{1} \approx$ $a_{3} / a_{1}<1$ ), with maximum projected ellipticity consistent with that of an E3 galaxy. For initially unstable models, the central density concentration drops during evolution, but the radial scale of the system remains approximately constant. For example, for the $(1 / 2 ; 3)$ model, the central concentration at the end of the simulation is more than 10 times smaller than that of the initial conditions, but the radius of the sphere that contains $5 \%$ of the total mass increases only by $6 \%$, while the half-mass radius remains basically unchanged.

We have studied the evolution of the unstable models in terms of exponential growth curves of the form

$g(t)=g_{0}+g_{1}[\exp (k t)-1]$

where $g_{0}, g_{1}$, and the growth rate $k$ are free parameters and $g$ represents a global quantity such as the global anisotropy ratio. To capture the initial, approximately linear phase of the evolution, we have decided to make the fit in terms of the exponential growth over a reduced time interval, from $t=0$ to $t=t_{\text {lin }}<t_{\text {end }}$, defined implicitly by the relation:

$\left(\frac{2 K_{\mathrm{r}}}{K_{\mathrm{T}}}\right)\left(t_{\text {lin }}\right)=\frac{1}{2}\left[\left(\frac{2 K_{\mathrm{r}}}{K_{\mathrm{T}}}\right)\left(t_{\text {end }}\right)+\left(\frac{2 K_{\mathrm{r}}}{K_{\mathrm{T}}}\right)(0)\right]$.

Obviously, this choice of $t_{\text {lin }}$ is arbitrary and should be considered only as a convenient reference time-scale.

The results of the simulations are reported in Table 1. The threshold for instability is found to be at $2 K_{\mathrm{r}} / K_{\mathrm{T}} \approx 1.70$, consistent with the criterion of Polyachenko \& Shukhman (1981). Close to conditions of marginal stability, quantities such as $2 K_{\mathrm{r}} / K_{\mathrm{T}}$ vary slowly and by very small amounts, so that the fit in terms of exponential growth curves is not well determined.

An interesting result is the following. We checked whether the final state reached as a result of the instability could be represented by a model of the $f^{(v)}$ family to which the initial unstable model belonged. To do this, we fitted the spherically 
Table 1. Growth rate $k$, with an estimate of the related uncertainty $\Delta k$, for the radial orbit instability from the simulation of a set of $f^{(v)}$ models. Here $\kappa=2 K_{\mathrm{r}} / K_{\mathrm{T}}$ is the global anisotropy parameter and $\eta=$ $a_{3} / a_{1}$ is the axis ratio of the inertia ellipsoid referred to the sphere of radius $3 r_{M}$. For $M=10^{11} M_{\odot}$ and $r_{M}=5 \mathrm{kpc}, k$ is given in units of $10^{-8} \mathrm{yr}^{-1}$.

\begin{tabular}{cccccc}
\hline \hline$(v ; \Psi)$ & $k$ & $\Delta k$ & $\kappa(0)$ & $\kappa\left(t_{\text {end }}\right)$ & $\eta\left(t_{\text {end }}\right)$ \\
\hline$(1 ; 2.0)$ & 0.85 & 0.1 & 2.65 & 1.87 & 0.65 \\
$(1 ; 3.0)$ & 0.48 & 0.07 & 2.17 & 1.84 & 0.67 \\
$(1 ; 3.2)$ & 0.37 & 0.07 & 2.09 & 1.84 & 0.69 \\
$(1 ; 3.4)$ & 0.34 & 0.07 & 2.02 & 1.83 & 0.74 \\
$(1 ; 3.6)$ & 0.30 & 0.05 & 1.95 & 1.85 & 0.80 \\
$(1 ; 3.8)$ & 0.16 & 0.04 & 1.89 & 1.82 & 0.85 \\
$(1 ; 4.0)$ & 0.08 & 0.05 & 1.84 & 1.81 & 0.91 \\
$(1 ; 4.2)$ & 0.007 & 0.10 & 1.78 & 1.76 & 0.94 \\
$(1 ; 5.0)$ & 0.001 & 0.01 & 1.60 & 1.60 & 0.99 \\
$(1 ; 9.0)$ & $<10^{-4}$ & - & 1.32 & 1.32 & 0.99 \\
$(3 / 8 ; 3.0)$ & 0.90 & 0.10 & 2.28 & 1.86 & 0.73 \\
$(3 / 8 ; 5.0)$ & 0.45 & 0.15 & 1.75 & 1.71 & 0.93 \\
$(1 / 2 ; 3.0)$ & 0.70 & 0.10 & 2.21 & 1.86 & 0.70 \\
$(1 / 2 ; 4.0)$ & 0.43 & 0.05 & 1.92 & 1.80 & 0.85 \\
$(1 / 2 ; 5.0)$ & 0.1 & 0.2 & 1.68 & 1.67 & 0.96 \\
$(1 / 2 ; 6.0)$ & $<10^{-3}$ & - & 1.54 & 1.54 & 0.99 \\
$(3 / 4 ; 3.0)$ & 0.64 & 0.05 & 2.20 & 1.85 & 0.70 \\
$(3 / 4 ; 4.0)$ & 0.16 & 0.02 & 1.86 & 1.76 & 0.84 \\
$(3 / 4 ; 5.0)$ & $<2 \times 10^{-2}$ & - & 1.63 & 1.63 & 0.98 \\
\hline & & & & &
\end{tabular}

averaged density and anisotropy profiles of the end-states of the simulations by means of the same family of $f^{(v)}$ models. The best fit model thus identified (note the quality of the fit in relation to both kinematics and density distribution) turns out to be the marginally stable model of the sequence with the same value of $v$ (see also the interesting arguments and results by Palmer et al. 1990). In the case of $v=1$, which we studied in greatest detail, the profiles of the end-states obtained starting from initially unstable models with $\Psi$ in the range from 3 to 4 are well fitted by the $(1 ; 4.2)$ model (see Fig. 12). For the violently unstable initial $(1 ; 2)$ model, the final state is best reproduced by the $(7 / 8 ; 4)$ model, which is still moderately unstable.

We have also run a number of simulations of models expected to be stable and checked that indeed the models preserve their state for many dynamical time scales. The most concentrated model that we have been able to simulate properly is the $(1 / 2 ; 9.4)$ model, for which we recall that the central concentration is $\rho(0) / \rho\left(r_{M}\right)=1.14 \times 10^{5}$. This also quantifies the kind of gradients and ranges of densities that our code is able to handle (see Fig. 13).

\section{A first comparison with the observations}

The results in terms of the $R^{1 / n}$ law described in Sect. 3.2 already show that the $f^{(v)}$ models possess realistic density profiles. This encouraged us to consider a direct comparison with
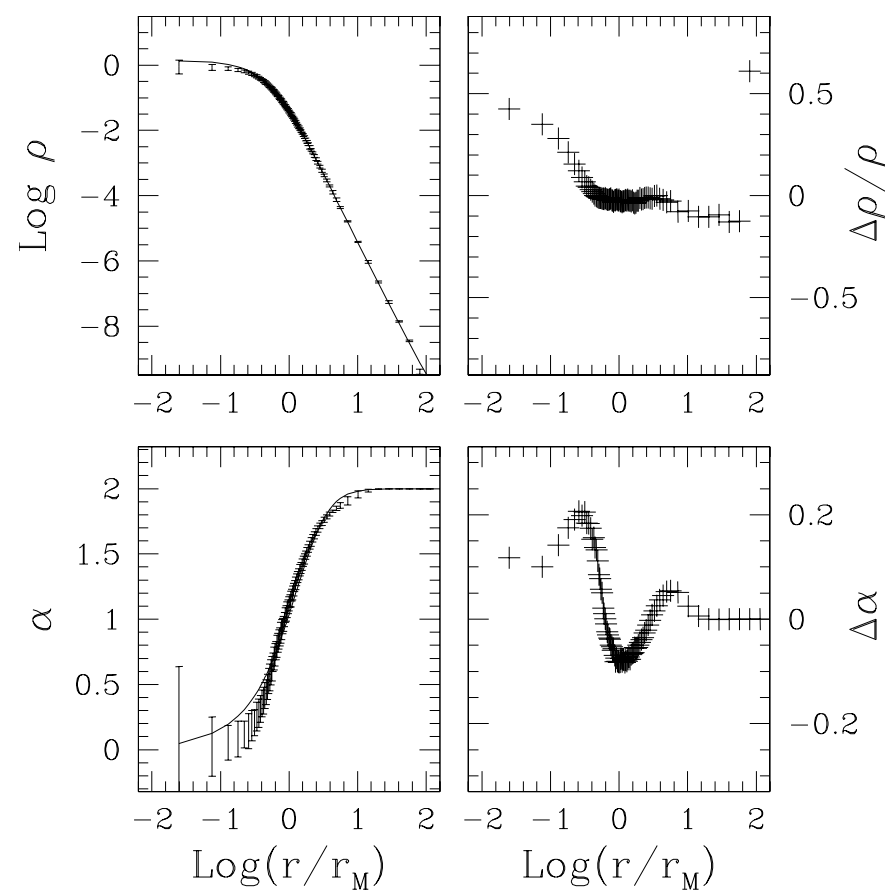

Fig. 12. On the left frames, density and anisotropy profiles of the endproducts of a simulation starting from the unstable $(1 ; 3.2)$ model (error bars) fitted by the marginally stable $(1 ; 4.2)$ model of the $f^{(v)}$ family (solid line); units for $\rho$ are the units adopted in the simulation. The right frames show the residuals $\Delta \rho=\rho^{(4.2)}-\rho$ and $\Delta \alpha=\alpha^{(4.2)}-\alpha$.

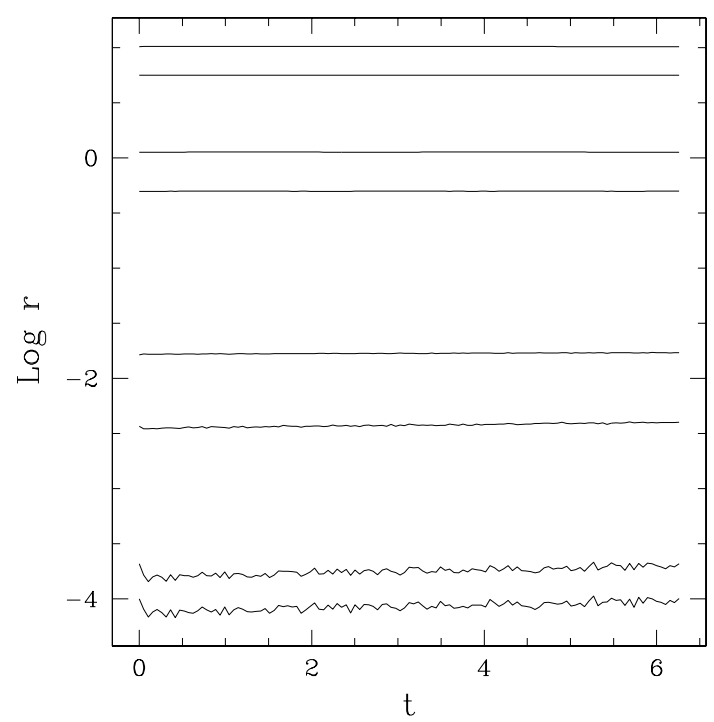

Fig. 13. Evolution of the fractional mass radii (of the sphere containing, from the bottom to the top, $6.25 \times 10^{-5}, 1.25 \times 10^{-4}, 0.01,0.05,0.5$, $0.7,0.95,0.99 \mathrm{M}$, respectively) for a simulation with $8 \times 10^{5}$ particles of the concentrated $f^{(v)}$ model $(1 / 2 ; 9.4)$. The model is stable over the time interval considered (about 10 dynamical times). The radius $r$ and the time $t$ are given in units of $10 \mathrm{kpc}$ and $10^{8} \mathrm{yr}$.

an observed galaxy. For the purpose, we picked the round elliptical galaxy NGC 3379, which apparently does not possess significant amounts of dark matter (Saglia et al. 1992; Romanowsky et al. 2003; note that the models discussed in the present paper are one-component models and thus are not applicable to systems with prominent dark halos). This galaxy 


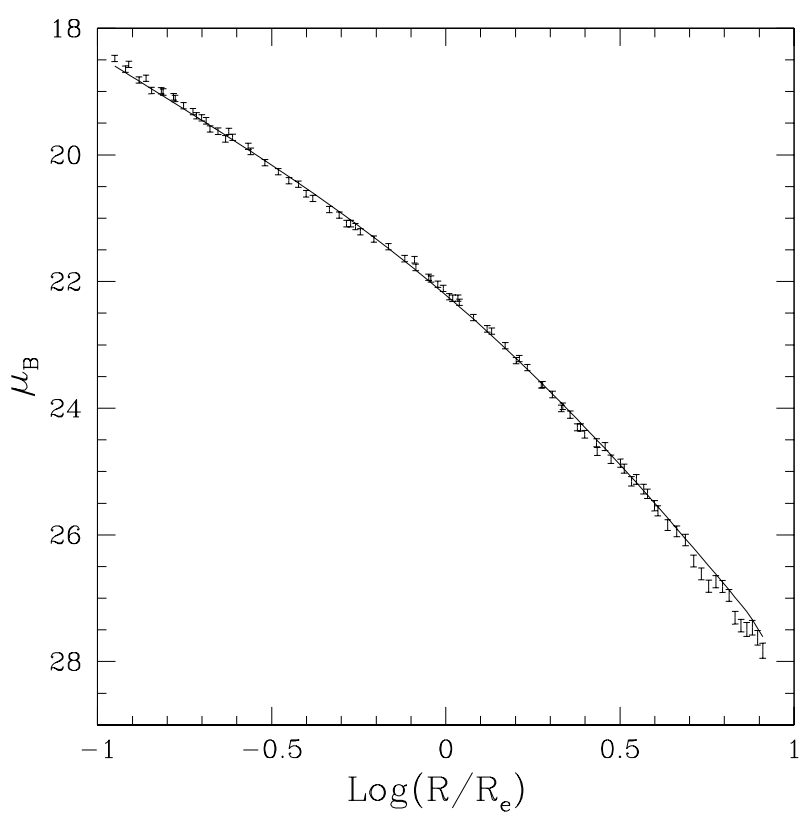

Fig. 14. Comparison between the photometric profile of NGC 3379 and the $(1 / 2 ; 9.4)$ model of the $f^{(v)}$ family. The photometric profile in $B$ is taken from de Vaucouleurs \& Capaccioli (1979). Other members of the family (e.g. the $(1 ; 9.2)$ model) could better reproduce the observed data especially at large radii, but they would perform less well on the velocity dispersion profile.

has an $R^{1 / 4}$ luminosity profile (de Vaucouleurs \& Capaccioli 1979; Capaccioli et al. 1990). For the kinematics, we considered the data of Statler \& Smecker-Hane (1999) and recently published data-points based on planetary nebulae that extend well beyond $R_{\mathrm{e}}$ (Romanowsky et al. 2003).

The $f^{(v)}$ model that best describes the data is shown in Figs. 14-15. From such model, by adopting a distance of $11 \mathrm{Mpc}$ for the galaxy and an absolute magnitude in $B$ band $M_{B}=-20.0$, we obtain a mass-to-light ratio $M / L_{B}=4.7$ in solar units. Population synthesis models for NGC 3379 predict a mass-to-light ratio between 4 and 9 (Gerhard et al. 2001). In comparison, Romanowsky et al. (2003) report a mass-to-light ratio $M / L_{B}=7.1 \pm 0.6$.

\section{Discussion and conclusions}

In this paper we have studied the structural and dynamical properties of a two-parameter family of models of partially relaxed stellar systems. These models had been proposed earlier (Stiavelli \& Bertin 1987) as physically relevant to the galaxy formation scenario based on collisionless collapse and incomplete violent relaxation. They had been noted to possess some realistic properties (for $v$ in the range 1/2-1 and for relatively large values of $\Psi$ ). However, they had been left basically aside and not studied further in systematic detail. Additional physical interest was noted recently (Bertin \& Trenti 2003), in an investigation focused on their thermodynamic properties, in relation to the paradigm of the gravothermal catastrophe. Because of such physical interest, we have decided to undertake a thorough comparison between the models and the products of collisionless collapse, as generated in $N$-body simulations. Such a

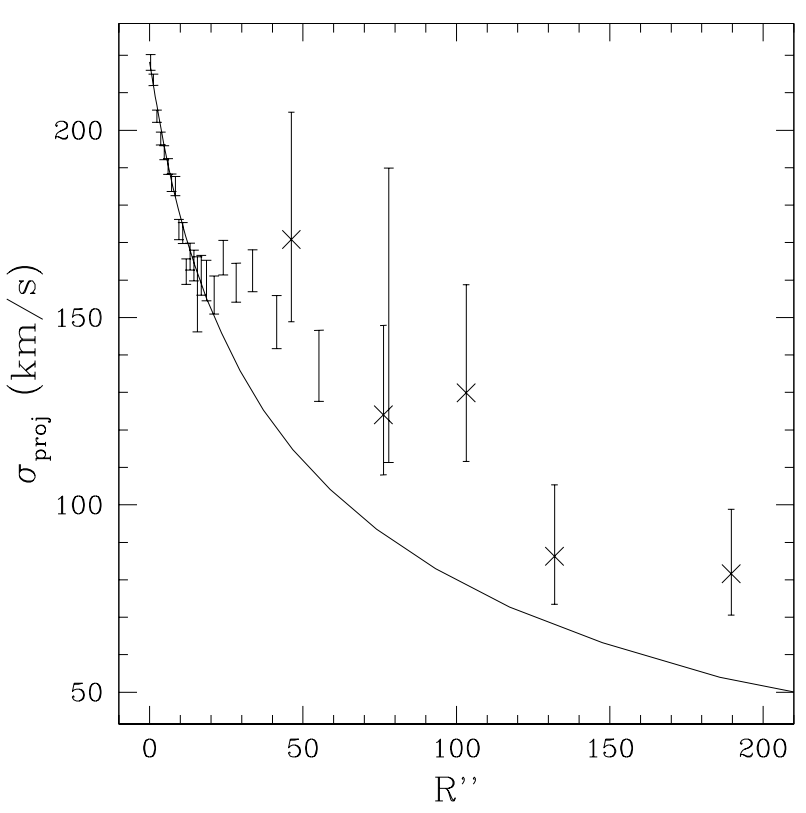

Fig. 15. Kinematic data for NGC 3379 described in terms of the $(1 / 2$; 9.4) model of the $f^{(v)}$ family. The inner data (plain error bars) are taken from the stellar spectroscopy of Statler \& Smecker-Hane (1999), while the outer data (crosses with error bars) refer to the binned velocity dispersion determined from the study of planetary nebulae (Romanowsky et al. 2003).

comparison will be the subject of a follow-up paper, of which the present article forms the necessary basis. In that paper we will consider a relatively large set of simulations of collisionless collapse, starting from a variety of initial conditions. We will address the issue of the conservation of $Q$ and identify the range of $v$ for which the global quantity is best conserved. Furthermore, we will show that in many cases the $f^{(v)}$ models can provide a surprisingly good fit to both the density and the pressure profiles, over nine orders of magnitude of the density distribution (see Bertin \& Trenti 2004). The best-fit models will turn out to be close to marginal stability with respect to the radial orbit instability.

Here we have shown that the family of $f^{(v)}$ models exhibits a variety of structural properties, within a common general behavior. The model characteristics can be summarized by referring to three separate regimes: low- $\Psi$ models (typically, $\Psi<4$ ), intermediate- $\Psi$ models (typically, $4<\Psi<8$ ), and high- $\Psi$ models (typically, $\Psi>8$ ). In practice, the values of $\Psi$ that mark the transition between different regimes do not depend significantly on the value of $v$, at least in the range explored in this paper (i.e., from $v=3 / 8$ to $v=1$ ).

In the intermediate- $\Psi$ and the high- $\Psi$ regimes the concentration ratio $\rho(0) / \rho\left(r_{M}\right)$ increases monotonically with $\Psi$; models with lower values of $v$ have larger values of $\rho(0) / \rho\left(r_{M}\right)$ (by up to one order of magnitude at given $\Psi$, in the explored range of $v$ ). In the same regimes, as $\Psi$ increases, the density distribution converges onto a common profile characterized by an approximate $r^{-4}$ behavior at $r>r_{M}$ and by an approximate $r^{-2}$ behavior at $r<r_{M}$, with an inner core that becomes smaller and smaller. In contrast, models in the low- $\Psi$ regime have a substantial core, comparable in structure to that of a Plummer 
or of an isochrone model. In general, the projected density distribution of the models is well fitted by the $R^{1 / n}$ law, with $n$ ranging from 2.5 to 8.5 . Typically, high- $\Psi$ models are accurately described in terms of the $R^{1 / 4}$ law.

The models are all characterized by significant radiallybiased pressure anisotropy. In terms of the global anisotropy ratio $2 K_{\mathrm{r}} / K_{\mathrm{T}}$, at given $\Psi$ models in the low- $\Psi$ regime have similar amounts of pressure anisotropy, which increases rapidly as $\Psi$ decreases. In practice, all low- $\Psi$ models should all be unstable with respect to the radial-orbit instability. Intermediate- $\Psi$ and high- $\Psi$ models appear to be safely stable, but they may be only marginally so for the lowest value of $v$ that we considered. This latter remark also explains why we have decided not to move below $v=3 / 8$, given the fact that radial pressure anisotropy is larger for lower- $v$ models. In terms of velocity dispersion profiles, to some extent pressure anisotropy is already significant at $r \approx 0.1 r_{M}$, even for stable models. However, out to $r \approx r_{M}$ the line profiles of stable models deviate very little from a Gaussian, with $h_{4}$ within the $5 \%$ level.

By means of an improved version of a code introduced earlier by van Albada, we have checked the properties of the models with respect to the radial-orbit instability, confirming the general expectations based on the global anisotropy parameter $2 K_{\mathrm{r}} / K_{\mathrm{T}}$. Curiously, we find that unstable models tend to evolve by staying close to the equilibrium sequence, while moving up in $\Psi$ so as to reach marginal stability. Furthermore, we have been able to show that the $N$-body code is able to handle highly concentrated models (up to $\Psi=9.4$, with $\left.\rho(0) / \rho\left(r_{M}\right)=1.14 \times 10^{5}\right)$, of which it has demonstrated the long-term stability.

Finally, a comparison with the observed surface brightness and kinematical profiles for the galaxy NGC 3379 has shown some merits and some limitations of the one-component family of $f^{(v)}$ models when applied to observed objects. In particular, it is interesting to see that, within the extremely idealized framework at the basis of the construction of these models (see also the general comments made in Sect. 1.1), the observed luminosity profile is very well reproduced over about ten magnitudes; at the same time the models can well reproduce the inner part of the relevant kinematical profile, inside $R_{\mathrm{e}}$. This comparison supports the view that the models studied in this paper may be helpful to describe the luminous component of elliptical galaxies. The obvious limitations of the one-component models that we are considering are clearly brought out by their inadequacy to capture the change in the observed kinematical profile occurring around $R_{\mathrm{e}}$ and thus their failure to reproduce such profile in the outer parts. This is interpreted as a signature of the presence of an additional component that our models, in the form developed so far, a priori ignore.

Acknowledgements. We would like to thank T. S. van Albada and P. Londrillo, for their collaboration and for a number of useful comments and suggestions, and N. Douglas and A. Romanowsky, for providing us with the data for NGC 3379. The interested reader is welcome to contact M. Trenti, who will be glad to provide the relevant numerical routines to initialize $N$-body simulations and to compute intrinsic and projected profiles for the $f^{(v)}$ family of models.

\section{Appendix A: Constants and scales}

From the definition of the $f^{(v)}$ distribution function (see Eq. (2)), the constants $(A, a, d)$ have the following dimensions:

$A=\left[M L^{-6} T^{3}\right]$,

$a=\left[L^{-2} T^{2}\right]$,

$d=\left[L^{-v / 2} T^{-v / 2}\right]$.

Therefore, as scales for mass and radius we may refer to:

$R_{\text {scale }}=a^{-1 / 4} d^{-1 / v}$,

$M_{\text {scale }}=A a^{-9 / 4} d^{-3 / v}$.

Then we introduce the dimensionless quantities $\hat{r}=r / R_{\text {scale }}$ (and thus $\left.\hat{r}_{M}=\hat{r}_{M}(v, \Psi)=r_{M} / R_{\text {scale }}\right), \hat{M}=\hat{M}(v, \Psi)=$ $M / M_{\text {scale }}$, where $\hat{M}(v, \Psi)=\int \hat{\rho}(\hat{r}) \mathrm{d}^{3} \hat{r}, \hat{w}=a^{1 / 2} w$, and $\hat{\Phi}=a \Phi$ for radius, mass, velocity, and potential, respectively.

Recalling the expression for $\gamma$ (see Sect. 2, after Eq. (2)), for given $v$ we can express $(A, a, d)$ in terms of $\left(M, r_{M}, \Psi\right)$ :

$a=\frac{1}{4 \pi G \gamma(v, \Psi)} \frac{r_{M}}{\hat{r}_{M}(v, \Psi)} \frac{\hat{M}(v, \Psi)}{M}$
$d=[4 \pi G \gamma(v, \Psi)]^{v / 4}\left(\frac{M}{\hat{M}(v, \Psi)}\right)^{v / 4}\left(\frac{\hat{r}_{M}(v, \Psi)}{r_{M}}\right)^{5 v / 4}$
$A=\frac{1}{[4 \pi G \gamma(v, \Psi)]^{3 / 2}}\left(\frac{\hat{r}_{M}(v, \Psi)}{r_{M}}\right)^{3 / 2}\left(\frac{\hat{M}(v, \Psi)}{M}\right)^{1 / 2}$

\section{References}

Abramowitz, M., \& Stegun, I. 1965, Handbook of Mathematical Functions (New York: Dover)

Antonov, V. A. 1962, Vestnik Leningr. Univ., No. 19, 96 (Engl. Transl.: in Structure and Dynamics of Elliptical Galaxies, ed. T. de Zeeuw (Dordrecht: Reidel), 1986, 531)

Berntsen, J. T. E., \& Genz, A. 1991, ACM Tran. Mat. Soft., 17, 437

Bertin, G., Ciotti, L., \& Del Principe, M. 2002, A\&A, 386, 149

Bertin, G., Pegoraro, F., Rubini, F., \& Vesperini, E. 1994, ApJ, 434, 94

Bertin, G., \& Stiavelli, M. 1984, A\&A, 137, 26

Bertin, G., \& Stiavelli, M. 1993, Rep. Progr. Phys., 56, 493

Bertin, G., \& Trenti, M. 2003, ApJ, 584, 729

Bertin, G., \& Trenti, M. 2004, AIP Conf. Proc. 703, ed. G. Bertin, D. Farina, R. Pozzoli (Melville, New York: American Institute of Physics), 318

Capaccioli, M., Held, E. V., Lorenz, H., \& Vietri, M. 1990, AJ, 99, 1813

Carollo, C. M., de Zeeuw, P. T., \& van der Marel, R. P. 1995, MNRAS, 276,1131

de Vaucouleurs, G. 1948, Annales d'Astrophysique, 11, 247

de Vaucouleurs, G., \& Capaccioli, M. 1979, ApJS, 40, 699

de Zeeuw, P. T., Bureau, M., Emsellem, E., et al. 2002, MNRAS, 329, 513

Fridman, A. M., \& Polyachenko, V. L. 1984, Physics of Gravitating Systems (Berlin: Springer-Verlag)

Gerhard, O. 1991, MNRAS, 250, 812

Gerhard, O. 1993, MNRAS, 265, 213

Gerhard, O., Kronawitter, A., Saglia, R. P., \& Bender, R. 2001, AJ, 121, 1936

Ghigna, S., Moore, B., Governato, F., et al. 2000, ApJ, 544, 616

Gonzalez-Garcia, C., \& van Albada, T. S. 2003, MNRAS, 342, 36 
Hénon, M. 1959, Annales d'Astrophysique, 22, 126

Hernquist, L. 1990, ApJ, 356, 359

Hjorth, J., \& Madsen, J. 1995, ApJ, 445, 55

Jaffe, W. 1983, MNRAS, 202, 995

Katz, J. 1978, MNRAS, 183, 765

Katz, J. 1979, MNRAS, 189, 817

Katz, J. 1980, MNRAS, 190, 497

Lanzoni, B., Ciotti, L., Cappi, A., Tormen, G., \& Zamorani, G. 2004, ApJ, 600, 640

Londrillo, P., Nipoti, C., \& Ciotti, L. 2003, Mem. Soc. Astron. It. Suppl., 1, 18

Lynden-Bell, D. 1967, MNRAS, 131, 101

Lynden-Bell, D., \& Wood, R. 1968, MNRAS, 138, 495

Merritt, D. 1985, AJ, 90, 1027

Merritt, D., Tremaine, S., \& Johnstone, D. 1989, MNRAS, 236, 829

Meza, A., Navarro, J. F., Steinmetz, M., \& Eke, V. R. 2003, ApJ, 590, 619

Moore, B., Governato, F. Quinn, T., Stadel, J., \& Lake, G. 1998, ApJ, 499,5

Navarro, J. F., Frenk, C. S., \& White, S. D. M. 1997, ApJ, 490, 593
Nipoti, C., Londrillo, P., \& Ciotti, L. 2003, MNRAS, 342, 501

Palmer, P. L., Papaloizou, J., \& Allen, A. J. 1990, MNRAS, 246, 415

Plummer, H. C. 1915, MNRAS, 76, 107

Polyachenko, V., \& Shukhman, I. 1981, SvA, 25, 533

Press W. H., Teukolsky S. A., Vetterling W. T., \& Flannery B. P. 1992, Numerical Recipes in C (UK: Cambridge University Press)

Romanowsky, A. J., Douglas, N. G., Arnaboldi, M., et al. 2003, Science, 301, 1696

Saglia, R. P., Bertin, G., \& Stiavelli, M. 1992, ApJ, 384, 433

Sersic, J. L. 1968, Atlas de galaxias australes, Observatorio Astronomico, Cordoba

Shu, F. 1978, ApJ, 225, 83

Shu, F. 1987, ApJ, 316, 502

Statler, T. S., \& Smecker-Hane, T. 1999, AJ, 117, 839

Stiavelli, M., \& Bertin, G. 1987, MNRAS, 229, 61

Trenti, M. 2004, Ph.D. Thesis, Scuola Normale Superiore, Pisa, in progress

van Albada, T. S. 1982, MNRAS, 201, 939

van Albada, T., S., \& van Gorkom, J. H. 1977, A\&A, 54, 121

Voglis, N. 1994, MNRAS, 267, 379 